\title{
Update on the endorsement of CONSORT by high impact factor journals: a survey of journal "Instructions to Authors" in 2014
}

\author{
Larissa Shamseer ${ }^{1,2^{*}}$, Sally Hopewell ${ }^{3}$, Douglas G. Altman ${ }^{3}$, David Moher ${ }^{1,2}$ and Kenneth F. Schulz ${ }^{4,5}$
}

\begin{abstract}
Background: The CONsolidated Standards Of Reporting Trials (CONSORT) Statement provides a minimum standard set of items to be reported in published clinical trials; it has received widespread recognition within the biomedical publishing community. This research aims to provide an update on the endorsement of CONSORT by high impact medical journals.

Methods: We performed a cross-sectional examination of the online "Instructions to Authors" of 168 high impact factor (2012) biomedical journals between July and December 2014. We assessed whether the text of the "Instructions to Authors" mentioned the CONSORT Statement and any CONSORT extensions, and we quantified the extent and nature of the journals' endorsements of these. These data were described by frequencies. We also determined whether journals mentioned trial registration and the International Committee of Medical Journal Editors (ICMJE; other than in regards to trial registration) and whether either of these was associated with CONSORT endorsement (relative risk and $95 \%$ confidence interval). We compared our findings to the two previous iterations of this survey (in 2003 and 2007). We also identified the publishers of the included journals.
\end{abstract}

Results: Sixty-three percent (106/168) of the included journals mentioned CONSORT in their "Instructions to Authors." Forty-four endorsers (42\%) explicitly stated that authors "must" use CONSORT to prepare their trial manuscript, $38 \%$ required an accompanying completed CONSORT checklist as a condition of submission, and $39 \%$ explicitly requested the inclusion of a flow diagram with the submission. CONSORT extensions were endorsed by very few journals. One hundred and thirty journals (77\%) mentioned ICMJE, and 106 (63\%) mentioned trial registration.

Conclusions: The endorsement of CONSORT by high impact journals has increased over time; however, specific instructions on how CONSORT should be used by authors are inconsistent across journals and publishers. Publishers and journals should encourage authors to use CONSORT and set clear expectations for authors about compliance with CONSORT.

Keywords: CONSORT, Trial, Reporting, Endorsement, Registration

\section{Background}

The CONsolidated Standards Of Reporting Trials (CONSORT) guideline was first published 20 years ago. Since that time, it has received widespread attention, including being lauded as a twentieth century milestone in health research methodology [1]. CONSORT is intended

\footnotetext{
* Correspondence: Ishamseer@ohri.ca

${ }^{1}$ Centre for Practice-Changing Research, Ottawa Hospital Research Institute, Ottawa, ON K1H 8L6, Canada

${ }^{2}$ School of Epidemiology, Public Health and Preventative Medicine, University of Ottawa, Ottawa, ON K1H 8M5, Canada

Full list of author information is available at the end of the article
}

for use as a guide to reporting essential components of trial methods and findings by those preparing and reviewing reports of randomized trials.

The uptake of CONSORT is reflected in a number of metrics. Combined, the 1996 [2], 2001 [3], and 2010 [4] publications of the CONSORT Statement and Explanation and Elaboration documents $[5,6]$ have been cited more than 12,000 times (according to Scopus, May 2015), making CONSORT among the most highly cited biomedical publications of all time. Perhaps a more reflective measure of CONSORT uptake, however, is the 
support from a number of major editorial organizations (i.e., the International Committee of Medical Journal Editors, Committee on Publication Ethics, and World Association of Medical Editors) and its endorsement by more than 600 biomedical journals. Endorsement of CONSORT is typically demonstrated by a statement in a journal's "Instructions to Authors" indicating support for CONSORT or a recommendation or requirement for authors to adhere to CONSORT when submitting a manuscript of a randomized trial for publication consideration.

A systematic review published in 2012 determined that journal endorsement of CONSORT was associated with more completely reported trials, based on assessments of more than 16,000 trials [7]. Four CONSORT checklist items (scientific rationale, sample size, sequence generation, and allocation concealment) and a summary score (including varying checklist items across studies reporting this) were significantly more likely to be reported in trials published in endorsing journals compared to their nonendorsing counterparts, and almost all remaining items were more completely reported, although this result was not statistically significant. However, reviews of publications also show that the reporting of key items remains well short of acceptable, with the reporting of details of random-allocation procedures being especially poor.

The extent to which journals are enforcing or checking for adherence to CONSORT in submitted/published trials is unknown and difficult to ascertain. To date, the best and most practical way to determine a journal's stance on the matter of endorsement is to identify whether journals make recommendations around CONSORT in their "Instructions to Authors." Two earlier studies have characterized CONSORT's endorsement across high impact factor biomedical journals over time. In 2003, $22 \%$ of high impact factor journals endorsed CONSORT [8] - this increased to $38 \%$ in 2007 [9]; however both studies concluded that many journals used ambiguous language in terms of what was meant by endorsement.

This study provides an up-to-date assessment of the extent and nature of CONSORT endorsement in high impact factor journals in 2014 (the first since publication of CONSORT 2010) and describes changes in endorsement over time.

\section{Methods}

\section{Selection of included journals}

Journals were selected using the strategy adopted in the previous studies [8, 9]. Briefly, this consisted of using 2012 impact factors (Thomson Reuters Journal Citation Reports-Science Citation Index Expanded) to select the top five impact factor journals for each of 33 medical specialties and the top 15 impact factor journals in general and internal medicine. Journals that either explicitly indicated or were found not to publish clinical trials, as determined after a PubMed search (or an inspection of the journal scope when unsure), were excluded and replaced by the next one on the list. Journals that appeared in more than one specialty were not replaced by another journal but were included only once in the analysis.

\section{Survey of the "Instructions to Authors" published by the journals}

One assessor (LS) examined the "Instructions to Authors" on the website of each included journal and extracted the information of interest for each journal between July and December 2014. Specifically, we extracted information on whether CONSORT and/or its extensions (for abstracts [10], acupuncture trials [11], cluster trials [12], harms outcomes [13], herbal interventions [14], noninferiority and equivalence trials [15], nonpharmacological interventions [16], patient-reported outcomes [17], and pragmatic trials [18]) were mentioned and, if so, assessed the following: whether CONSORT or any extension was mentioned as a "requirement" or as a "recommendation" or was unclear; which version of CONSORT (1996 [2], 2001 [3, 5], 2010 [4, 6]) was referenced, if any; and which CONSORT document (website, Statement paper, Explanatory paper, checklist, flow diagram, or other) was referred to, if any. If journals "required" CONSORT as a condition of submission, we extracted whether a flow diagram or checklist was an explicit requirement. While we aimed to examine endorsement of any official CONSORT extensions, we recognize that some types of trials for which CONSORT extensions exist (e.g., acupuncture or herbal interventions) may be published in niche journals not included in our sample [19]. In accordance with the original search strategy for this study, we made no specific attempt to identify/include such journals.

Any mention of ICMJE and reference to clinical trial registration ("recommended" or "required") were also sought and extracted. Identification of the publisher of the included journals was also sought.

\section{Analysis}

Data were summarized descriptively using frequencies. We examined whether any mention of ICMJE and trial registration was associated with CONSORT endorsement (relative risk and $95 \%$ confidence interval) and summarized the journal endorsement status for publishers with more than one journal included in our sample. We present our data together with findings from previous iterations of this study [8,9], and we compared the stability of the endorsement status for journals that appeared in more than one study year. 


\section{Results}

One hundred and eighty journals were identified in Thomson Reuters' Journal Citation Reports database, of which, 12 were duplicates; thus, 168 journals were included in our sample.

\section{CONSORT endorsement}

Of the 168 journals, 106 (63\%) mentioned CONSORT in their online "Instructions to Authors," compared to $38 \%(62 / 165)$ in 2007 and $22 \%(36 / 166)$ in 2003. This is a relative increase of $66 \%$ since 2007 and $186 \%$ since 2003. Eighty-nine journals were included in all 3 years of the study; of these, 26 (29\%) became endorsers between 2003 and 2007, and 18 (20\%) endorsed CONSORT sometime between 2007 and 2014. No journals reversed their endorsement across the study years.

While 44 journals (42\%) required authors to use CONSORT, and 56 journals (53\%) recommended its use, fewer journals stated that submission of a completed checklist $(n=38)$ or a flow diagram $(n=39)$ was required as a condition of submission (Table 1). The 44 journals requiring authors to use CONSORT used explicit language, e.g. "Authors of trials must adhere to the CONSORT reporting guidelines appropriate to their trial design," or "before the trial can undergo peer review, authors must provide a completed CONSORT checklist as a supporting file." Journals recommending CONSORT $(n=56)$ used less forceful language, e.g. "Authors should adhere to these guidelines when drafting their manuscript." In six journals, CONSORT was mentioned, but the extent of the journal's recommendation was unclear, e.g., "Editorial assistance includes, but is not limited to, providing specific guidance regarding transparent reporting of items mentioned in pertinent reporting standards (e.g., CONSORT, PRISMA)." Seventy-three journals (69 \%) referred to the CONSORT checklist, and 57 (54\%) referred to the flow diagram. In addition, while we did not set out to collect this information, we noted that several journals informed authors of how the checklist would be used during the peer review process; for example, "peer reviewers will be asked to refer to these checklists when evaluating such studies."

The CONSORT website, which launched in 2005, was mentioned in the "Instructions to Authors" in 46 (58 \%) of the endorsing journals in 2007 compared to being mentioned in 84 (79\%) of the endorsers in 2014. Eighteen (17\%) of 106 endorsing journals referenced the most up-to-date version of CONSORT (CONSORT 2010), 11 (10 \%) referenced the CONSORT 2001 Statement, and two $(2 \%)$ cited the original version of CONSORT (1996). Indeed, among journals mentioning CONSORT, $83 \%$ did not reference the most current CONSORT Statement. No endorsing journals referred either to the 2001 or 2010 CONSORT Explanatory documents. Seventy-five (71 \%) of the endorsing journals did not reference any CONSORT publication. Nine journals $(8 \%)$ referred to both the website and the CONSORT Statement. Five journals (5 \%) did not refer to any specific CONSORT document or to the website.

\section{CONSORT extension endorsement}

Only 22 of the 168 included journals (13\%) mentioned any of the nine CONSORT extensions published at the time of searching, of which, all except one also endorsed CONSORT (Table 2). No journals in our sample endorsed the CONSORT extension for acupuncture interventions [11]. Of note, the Abstracts and Harms extensions were explicitly incorporated into the CONSORT 2010 checklist.

\section{ICMJE and trial registration}

One hundred thirty journals (77 \%) mentioned ICMJE in their "Instructions to Authors" in 2014, a large increase from $42 \%(n=69 / 165)$ of the journals in 2007 and $43 \%$ $(n=72 / 166)$ in 2003 (Table 1). One hundred seventeen (90\%) provided a link to the ICMJE website, 40 (34 \%) of which also mentioned the most recent ICMJE guidance [20]. While $59(50 \%)$ cited an older version of the ICMJE recommendations, most (92\%) also provided a link to the ICMJE website where the most up- to-date documents are hosted. Four further journals (3\%) referenced specific ICMJE recommendations (e.g., "ICMJE criteria for authorship") but provided no citation or link to an ICMJE document. Three journals (2\%) mentioned ICMJE but did not provide any link or reference. Journals that referred to CONSORT were more likely to refer to ICMJE in the "Instructions to Authors" (97/106, $92 \%)$ than those journals not referring to CONSORT (33/62, 53 \%) (relative risk 1.72, $95 \%$ confidence interval [CI] 1.35 to 2.19 ).

Of the 168 journals in the sample, 106 (63\%) mentioned trial registration (CONSORT 2010 checklist item 23) in their "Instructions to Authors" (Table 1); 78 (74 \%) required registration of submitted trials, 25 (24\%) recommended registration, and three made no specific statement of support for registration. Furthermore, 27/106 journals (35\%) referred only to the ICMJE statement about trial registration (which references at least seven registry options) [21], 9/106 (8\%) referred only to clinicaltrials.gov, $12 / 106$ (11\%) referred only to the World Health Organization Clinical Trials Platform [22], and 41/106 (39\%) journals referred to a combination of these three. Thirteen journals (12\%) mentioned trial registration but did not specifically mention or provide a link to one or more trial registries. Journals mentioning CONSORT were more likely to mention trial registration $(91 / 106,86 \%)$ than those not mentioning 
Table 1 Mentions of CONSORT, ICMJE, and trial registration in the "Instructions to Authors" from the journals

\begin{tabular}{|c|c|c|c|c|}
\hline & & 2014 & $2007^{2}$ & $2003^{3}$ \\
\hline & & (2012 IF) & (2006 IF) & (2001 IF) \\
\hline & & $N=168(\%)$ & $N=165(\%)$ & $N=166(\%)$ \\
\hline CONSORT Statement & & $106(63 \%)$ & $62(38 \%)$ & $36(22 \%)$ \\
\hline & Required & $44(42 \%)$ & $23(37 \%)$ & $8^{\mathrm{a}}$ \\
\hline & Recommended & $56(53 \%)$ & $39(63 \%)$ & 26 \\
\hline & Can't tell & $6(6 \%)$ & - & 2 \\
\hline & Submit with checklist & $38(36 \%)$ & $17(27 \%)$ & $\mathrm{nc}$ \\
\hline & Submit with flow diagram & $39(37 \%)$ & $\mathrm{nc}$ & $\mathrm{nc}$ \\
\hline Web address & & $84(79 \%)$ & $46^{\mathrm{b}}(58 \%)$ & $15+1^{c}(44 \%)$ \\
\hline Checklist & & $73(69 \%)$ & $\mathrm{nc}$ & $\mathrm{nc}$ \\
\hline Flow diagram & & $57(54 \%)$ & $\mathrm{nc}$ & $\mathrm{nc}$ \\
\hline 2010 Statement & & $18(17 \%)$ & $\mathrm{nr}$ & $\mathrm{nr}$ \\
\hline 2010 E\&E & & $0(0 \%)$ & $\mathrm{nr}$ & $\mathrm{nr}$ \\
\hline 2001 Statement & & $11(10 \%)$ & $18(29 \%)$ & $16(44 \%)$ \\
\hline $2001 \mathrm{E} \& \mathrm{E}$ & & $0(0 \%)$ & $1(2 \%)$ & $3(8 \%)$ \\
\hline 1996 Statement & & $2(2 \%)$ & $6^{d}(10 \%)$ & $9^{d}(17 \%)$ \\
\hline No specific reference/document indicated & & $5(5 \%)$ & $0(0 \%)$ & $0(0 \%)$ \\
\hline ICMJE & & 130 (77\%) & $69(42 \%)$ & $72(43 \%)$ \\
\hline Web address & & $117(90 \%)$ & $48(70 \%)$ & $23(40 \%)$ \\
\hline Up-to-date reference ${ }^{* *}$ & & $41(32 \%)$ & $3(4 \%)$ & $27(38 \%)$ \\
\hline Obsolete reference & & $59(45 \%)$ & $15(22 \%)$ & $41(57 \%)$ \\
\hline Other & & $4(3 \%)$ & $\mathrm{nc}$ & $\mathrm{nc}$ \\
\hline No specific reference & & $3(2 \%)$ & $6(9 \%)$ & $4(6 \%)$ \\
\hline Trial registration & & $106(63 \%)$ & $61(37 \%)$ & nc \\
\hline & Required & $78(74 \%)$ & $44(72 \%)$ & $\mathrm{nc}$ \\
\hline & Recommended & $25(24 \%)$ & $17(28 \%)$ & - \\
\hline & Can't tell & $3(3 \%)$ & - & - \\
\hline ICMJE (only) & & $27(25 \%)$ & $23(38 \%)$ & $\mathrm{nc}$ \\
\hline ClinicalTrials.gov (only) & & $9(8 \%)$ & $9(15 \%)$ & $\mathrm{nc}$ \\
\hline WHO International Clinical Trial Portal (only) & & $12(11 \%)$ & $4(7 \%)$ & $\mathrm{nc}$ \\
\hline Cites combination of the above & & $41(39 \%)$ & $10(16 \%)$ & - \\
\hline Other registry & & $3(3 \%)$ & - & - \\
\hline None & & $14(13 \%)$ & - & - \\
\hline
\end{tabular}

IF impact factor, CONSORT Consolidated Standards of Reporting Trials, $n c$ not collected, $n r$ not relevant, E\&E Explanation and Elaboration, ICMJE International Committee of Medical Journal Editors, WHO World Health Organization

${ }^{1}$ Bolded rows are the denominator for the numbers immediately following

2Data from Hopewell et al. [9]

${ }^{3}$ Data from Altman [8]

${ }^{a}$ Collapsed from Altman [8]: Required: required (3), must (5). Recommended: should (18), strongly encouraged (1), encouraged (2), recommended (2), please (2), may wish to consider (1). Can't tell: see (1) and no directive comment (1).

${ }^{\mathrm{b}}$ Web address was misspelled $(n=2)$

'Reference to JAMA website

${ }^{\mathrm{d}}$ Reference to an article citing the 1996 CONSORT Statement $(n=1)$

*Language ambiguous

"For 2014, this was any clear reference to the "Recommendations for the conduct, reporting, editing, and publication of scholarly work in medical journals": for 2007 and 2003 this was a > 2000 word article entitled "Uniform requirements for manuscripts submitted to biomedical journals: writing and editing for biomedical publication" 
Table 2 Mention of the CONSORT extensions published before December 2014 in "Instructions to Authors" on journal websites

\begin{tabular}{|c|c|}
\hline & 2014 \\
\hline & (2012 Impact factor) \\
\hline & $N=168(\%)$ \\
\hline Abstracts Extension (2008) & $11(7 \%)$ \\
\hline Required & 0 \\
\hline Recommended & 10 \\
\hline Can't tell & 1 \\
\hline Submit with checklist & 0 \\
\hline $\begin{array}{l}\text { Acupuncture Extension (STRICTA) (2001, updated } \\
\text { 2010) }\end{array}$ & $0(0 \%)$ \\
\hline Required & 0 \\
\hline Recommended & 0 \\
\hline Can't tell & 0 \\
\hline Submit with checklist & 0 \\
\hline Cluster Trials Extension (2004, updated 2012) & $11(7 \%)$ \\
\hline Required & 4 \\
\hline Recommended & 4 \\
\hline Can't tell & 3 \\
\hline Submit with checklist & 0 \\
\hline Harms Extension (2004) & $9(5 \%)$ \\
\hline Required & 5 \\
\hline Recommended & 1 \\
\hline Can't tell & 3 \\
\hline Submit with checklist & 0 \\
\hline Herbal interventions Extension (2006) & $2(1 \%)$ \\
\hline Required & 0 \\
\hline Recommended & 1 \\
\hline Can't tell & 1 \\
\hline Submit with checklist & 0 \\
\hline Noninferiority Extension (2006, updated 2012) & $4(2 \%)$ \\
\hline Required & 0 \\
\hline Recommended & 3 \\
\hline Can't tell & 1 \\
\hline Submit with checklist & 0 \\
\hline Nonpharmacological Extension (2008) & $4(2 \%)$ \\
\hline Required & 1 \\
\hline Recommended & 1 \\
\hline Can't tell & 2 \\
\hline Submit with checklist & 1 \\
\hline Pragmatic Trials Extension (2008) & $2(1 \%)$ \\
\hline Required & 0 \\
\hline Recommended & 2 \\
\hline
\end{tabular}

Table 2 Mention of the CONSORT extensions published before December 2014 in "Instructions to Authors" on journal websites (Continued)

\begin{tabular}{ll}
\hline Can't tell & 0 \\
Submit with checklist & 0 \\
Patient Reported Outcomes Extension (2013) & $1(0.6 \%)$ \\
Required & 0 \\
Recommended & 0 \\
Can't tell & 1 \\
Submit with checklist & 0 \\
\hline
\end{tabular}

CONSORT (15/62, 24 \%) (relative risk 3.55; 95 \% CI 2.27 to 5.55).

\section{CONSORT endorsement across publishers of high impact journals}

The 168 included journals were published by 39 publishers, 14 of whom published more than one of the journals. The ratio of endorsing journals to nonendorsing journals was inconsistent across journals (Table 3 ). In our sample, only one publisher with more than five journals included in the sample (the American Medical Association) had all endorsing journals and no nonendorsing journals.

Table 3 CONSORT endorsement status by publishers with more than one journal included in the sample

\begin{tabular}{lll}
\hline Publisher Name & $\begin{array}{l}\text { \# of journals included } \\
\text { in sample }\end{array}$ & $\begin{array}{l}\text { Endorsers: } \\
\text { Nonendorsers }\end{array}$ \\
AMERICAN DIABETES & 2 & $1: 1$ \\
ASSOCIATION & & $6: 0$ \\
AMERICAN MEDICAL & 6 & $0: 2$ \\
ASSOCIATION & & \\
AMERICAN PHYSIOLOGICAL & 2 & $2: 0$ \\
SOCIETY & & \\
AMERICAN SOCIETY & 2 & $2: 0$ \\
OF NEPRHOLOGY & & \\
AMERICAN SOCIETY & 2 & $3: 1$ \\
OF NUTRITION & & $4: 1$ \\
BIOMED CENTRAL & 4 & $28: 15$ \\
BMJ PUBLISHING GROUP & 5 & $15: 5$ \\
ELSEVIER & 43 & $0: 2$ \\
LIPPINCOTT WILLIAMS \& WILKINS & 20 & $6: 4$ \\
MARY ANN LIEBERT & 2 & $3: 7$ \\
NATURE PUBLISHING GROUP & 10 & $3: 0$ \\
OXFORD UNIVERSITY PRESS & 10 & $2: 0$ \\
PUBLIC LIBRARY OF SCIENCE & 3 & $1: 4$ \\
SAGE PUBLICATIONS & 2 & $15: 12$ \\
SPRINGER & 5 & \\
WILEY-BLACKWELL & 27 &
\end{tabular}




\section{Discussion}

The CONSORT Statement continues to gain traction among the biomedical journal community. As of December 2014, CONSORT was endorsed by $63 \%$ of high impact journals - almost triple the number of endorsers since the first investigation of this kind was conducted in 2003 and a $63 \%$ (relative) increase since 2007. While less than $20 \%$ of the endorsing journals referenced the most up-to-date CONSORT Statement and none referred to either of the CONSORT Explanation and Elaboration papers, most $(90 \%)$ provide a link to the CONSORT website, which always provides access to the most recent CONSORT documents. Recently, we have seen that journal endorsement of CONSORT is associated with more completely reported trials compared to nonendorsement, based on a 2012 systematic review including over 16,000 trials [7].

These numbers are encouraging. However, while CONSORT is a set of standard reporting recommendations, its implementation is far from standardized. For example, many of the endorsing journals in our sample did not make strong statements about CONSORT; approximately one half of the journals stop short of insisting that authors follow the guideline. Approximately one quarter of the journals ask that both a checklist and flow diagram are included with the trial submission, one third of the journals ask authors to submit either one or the other, and approximately one half of the endorsing journals do not specifically ask or require authors to submit either a CONSORT checklist or flow diagram. Even among trials published in endorsing journals in the aforementioned review, the reporting of many CONSORT items is still poor [7]. Undoubtedly, authors may be unaware or confused, about journal expectations regarding author use and adherence to CONSORT. This is in agreement with findings from the aforementioned review, which indicated that seemingly large relative effects of endorsement did not always translate into similar absolute effects [7]. For instance, the review found that the description of allocation concealment is complete in $81 \%$ more trials published in endorsing journals than in nonendorsing journals [7]. In absolute terms, this equated to only $45 \%$ of the trials in the endorsing journals $(n=876)$ and $22 \%$ of the trials published in the nonendorsing journals $(n=1,520)$. Given that allocation concealment is essential to maintaining trial validity, the fact that it is incompletely described in more than one half of trials is a serious concern. Therefore, a simple statement in a journal's "Instructions to Authors" about using or adhering to CONSORT is likely not sufficient on its own for improving reporting to the extent needed for some checklist items.

Authors can and should do better. Likewise, peer reviewers need to recognize the importance of identifying deficiencies in reporting. A 2014 series in The Lancet called for increased value and reduced waste in research. The authors called on gatekeepers of the publishing and dissemination process, including journals, to help authors increase their awareness, capacity, and capabilities around optimal reporting practices [23, 24]. Journals should be assured that their efforts toward CONSORT endorsement leading to better trial reporting are worthwhile. However, a supporting statement in a journal's "Instructions to Authors" is proving insufficient to improve trial reporting on a large scale, and more must be done. One study compared the reporting of trial abstracts in journals with varying levels of endorsement of the CONSORT for abstracts extensions [25]. It found that trials published in journals with a policy to actively implement the guideline (e.g., to send an email to authors to revise the abstract according to the guideline) were more completely reported than those in journals with no active policy [25].

In Box 1, we offer some unambiguous standard language that can be used by journals in their "Instructions to Authors" to demonstrate their support for CONSORT and indicate their expectations of the authors.

Collectively, the biomedical publishing industry is an important gatekeeper, determining which research reaches healthcare professionals and, ultimately, which is incorporated into patient-care decisions. The endorsement status of journals across publishers (Table 3) suggests that journals with the same publisher do not necessarily share a common set of "Instructions to Authors." A standard set of "Instructions to Authors" across publishers, allowing tailored information for each journal as necessary, may help to ensure better author understanding of journal expectations (including those around reporting) and facilitate an efficient peer review process by providing consistent messaging about journal expectations. Doing so may also benefit publishers. In this industry, the inexorable pursuit of impact metrics continues. Publishing research that adheres to minimum reporting standards,

Box 1. Recommended endorsement text for journals to include in their "Instructions to Authors"

"[Journal name] requires a completed CONSORT 2010 checklist and flow diagram as a condition of submission when reporting the results of a randomized trial. Templates for these can be found here or on the CONSORT website [www.consort-statement.org], which also describes several CONSORT checklist extensions for different designs and types of data beyond two-group parallel trials. At minimum, your article should report the content addressed by each item on the checklist. Meeting these basic reporting requirements will greatly improve the value of your trial report, may facilitate and/or enhance the peer review process, and may enhance the chance for eventual publication of your report." 
such as those outlined in CONSORT, will produce more usable reports. For publishers, increasing the usability of a report may increase its ability to be included, and therefore cited, in future research.

Lastly, our survey sampled journals with the highest 2012 impact factors in the medical specialties and in general medicine. Almost all of the general medical journals included in our sample (13/15) endorse CONSORT, and most have been involved in efforts to improve research reporting quality and completeness over the past two decades. Some specialties/subspecialties are now taking on the responsibility of improving research reporting within their specialty using a top-down approach. For example, a 2014 editorial in the Archives of Physical Medicine and Rehabilitation outlining a commitment to enforce mandatory compliance with reporting guidelines, including CONSORT, has been co-published by at least 35 journals, mostly within the rehabilitation specialty. This creates a complete "circle" of endorsing journals within a subspecialty, so authors must comply if they want to publish their research in a relevant journal [26]. This top-down model within a subspecialty is being repeated elsewhere [27, 28].

\section{Conclusions}

Many journals now endorse the CONSORT Statement, and such endorsement has been found to be associated with more complete reporting. Publishers and journals should encourage authors to use CONSORT and set clear expectations for authors about compliance with $\mathrm{CON}$ SORT (and indeed other reporting guidelines). They should make such information transparent and unambiguous in their "Instructions to Authors." Doing so will help authors to prepare high-quality reports of randomized trials, leading to better quality evidence to inform the treatment and prevention of disease.

\section{Abbreviations}

CONSORT, Consolidated Standards of Reporting Trials; ICMJE, International Committee of Medical Journal Editors

\section{Acknowledgements}

The authors would like to thank James Galipeau for assisting with extraction of two data items and Justin Thielman for his help extracting data for an earlier version of this project for presentation.

\section{Funding}

This study was carried out as part of operational research funding for CONSORT provided by the Medical Research Council in the United Kingdom; during the study period, salaries for $\mathrm{LS}$ and $\mathrm{SH}$ were supported by this funding. DGA, DM, and KFS form the CONSORT Executive group. DGA is an NIHR Senior Investigator. DM holds a University of Ottawa Research Chair.

\section{Availability of data and materials}

The dataset supporting the conclusions of this article is available in the Open Science Framework repository: https://osf.io/xb3jv/

\section{Authors' contributions}

DGA conceived the original study leading to this update, with additional methods conceived by SH. LS lead the data collection, analysis, and interpretation for this study and wrote the first draft of this manuscript. All authors (LS, SH, DGA, DM, and KFS) were involved with revising the manuscript for intellectual content and approved the final manuscript before submission. All authors read and approved the final manuscript.

\section{Competing interests}

DGA is the Co-Editor-in-Chief of Trials and KFS is on the Trials editorial board. Neither author had any role in the publication consideration of this manuscript.

\section{Author details}

${ }^{1}$ Centre for Practice-Changing Research, Ottawa Hospital Research Institute, Ottawa, ON K1H 8L6, Canada. ${ }^{2}$ School of Epidemiology, Public Health and Preventative Medicine, University of Ottawa, Ottawa, ON K1H 8M5, Canada. ${ }^{3}$ Centre for Statistics in Medicine, Nuffield Department of Orthopaedics, Rheumatology and Musculoskeletal Sciences, University of Oxford, Oxford OX3 7LD, UK. ${ }^{4}$ International Clinical Sciences Support Center, FHI 360, Durham, NC 27713, USA. ${ }^{5}$ Department of Obstetrics and Gynecology, UNC School of Medicine, Chapel Hill, NC 27599, USA.

Received: 26 February 2016 Accepted: 4 May 2016

Published online: 24 June 2016

\section{References}

1. Gabriel SE, Normand ST. Getting the methods right - the foundation of patient-centered outcomes research. N Engl J Med. 2012;367(9):787-90.

2. Begg C, Cho M, Eastwood S, Horton R, Moher D, Olkin I, et al. Improving the quality of reporting of randomized controlled trials. The CONSORT statement. J Am Med Assoc. 1996;276(8):637-9.

3. Moher D, Schulz KF, Altman DG. The CONSORT statement: revised recommendations for improving the quality of reports of parallel-group randomized trials. JAMA. 2001;285(15):1987-91.

4. Schulz KF, Altman DG, Moher D. CONSORT 2010 statement: updated guidelines for reporting parallel group randomised trials. BMJ. 2010;340:c332.

5. Altman DG, Schulz KF, Moher D, Egger M, Davidoff F, Elbourne D, et al. The revised CONSORT statement for reporting randomized trials: explanation and elaboration. Ann Intern Med. 2001;134(8):663-94.

6. Moher D, Hopewell S, Schulz KF, Montori V, Gøtzsche PC, Devereaux PJ, et al. CONSORT 2010 explanation and elaboration: updated guidelines for reporting parallel group randomised trials. BMJ. 2010;340:c869.

7. Turner L, Shamseer L, Altman DG, Weeks L, Peters J, Kober T, et al. Consolidated standards of reporting trials (CONSORT) and the completeness of reporting of randomised controlled trials (RCTs) published in medical journals. Cochrane Database Syst Rev. 2012;11(11):MR000030.

8. Altman DG. Endorsement of the CONSORT statement by high impact medical journals: survey of instructions for authors. BMJ. 2005;330(7499):1056-7.

9. Hopewell S, Altman DG, Moher D, Schulz KF. Endorsement of the CONSORT Statement by high impact factor medical journals: a survey of journal editors and journal 'Instructions to Authors'. Trials. 2008;9:20.

10. Hopewell S, Clarke M, Moher D, Wager E, Middleton P, Altman DG, et al. CONSORT for reporting randomized controlled trials in journal and conference abstracts: explanation and elaboration. PLoS Med. 2008:5(1):e20.

11. MacPherson H, Altman DG, Hammerschlag R, Youping L, Taixiang W, White A, et al. Revised STandards for Reporting Interventions in Clinical Trials of Acupuncture (STRICTA): extending the CONSORT statement. PLOS Med. 2010;7(6):e1000261.

12. Campbell MK, Piaggio G, Elbourne DR, Altman DG, The CONSORT Group. CONSORT 2010 statement: extension to cluster randomised trials. BMJ. 2012:345:e5661-1.

13. loannidis J, Evans SJW, Gøtzsche PC, O'Neill RT, Altman DG, Schulz KF, et al. Better reporting of harms in randomized trials: an extension of the CONSORT statement. Ann Intern Med Am Coll Physicians. 2004;141(10):781.

14. Gagnier JJ, Boon H, Rochon P, Moher D, Barnes J, Bombardier C. Recommendations for reporting randomized controlled trials of herbal interventions: Explanation and elaboration. J Clin Epidemiol. 2006:59(11):1134-49.

15. Piaggio G, Elbourne DR, Pocock SJ, Evans SJW, Altman DG. Reporting of noninferiority and equivalence randomized trials: extension of the CONSORT 2010 Statement. JAMA. 2012;308(24):2594-604.

16. Boutron I, Moher D, Altman DG, Schulz KF, Ravaud P. The CONSORT Group. Extending the CONSORT statement to randomized trials of 
nonpharmacologic treatment: explanation and elaboration. Ann Intern Med. 2008;148(4):295-309.

17. Calvert M, Blazeby J, Altman DG, Revicki DA, Moher D, Brundage MD. Reporting of patient-reported outcomes in randomized trials. The CONSORT PRO extension JAMA. 2013;309(8):814-22.

18. Zwarenstein M, Treweek S, Gagnier JJ, Altman DG, Tunis S, Haynes B, et al. Improving the reporting of pragmatic trials: an extension of the CONSORT statement. BMJ. 2008;337:a2390.

19. Pittler M, Abbot N, Harkness E, Ernst E. Location bias in controlled clinical trials of complementary/alternative therapies. J Clin Epidemiol. 2000:53(5):485-9.

20. ICMJE. Recommendations for the Conduct, Reporting, Editing, and Publication of Scholarly Work in Medical Journals. 2013. http://www.icmje. org/icmje-recommendations.pdf. Accessed 7 Jun 2016.

21. ICMJE. Trial Registration statement. http://www.icmje.org/about-icmje/fags/ clinical-trials-registration/. Accessed 20 April 2014.

22. WHO. Clinical Trial Portal. http://www.who.int/ictrp/network/primary/en/ Accessed 20 April 2014.

23. Macleod MR, Michie S, Roberts I, Dirnagl U, Chalmers I, loannidis JP, et al. Biomedical research: increasing value, reducing waste. Lancet. 2014; 383(9912):101-4.

24. Glasziou P, Altman DG, Bossuyt P, Boutron I, Clarke M, Julious S, et al. Reducing waste from incomplete or unusable reports of biomedical research. Lancet. 2014;383(9913):267-76.

25. Hopewell S, Ravaud P, Baron G, Boutron I. Effect of editors' implementation of CONSORT guidelines on the reporting of abstracts in high impact medical journals: interrupted time series analysis. BMJ. 2012;344:e4178.

26. Chan L, Heinemann A, Roberts J. Elevating the quality of disability and rehabilitation research: mandatory use of the reporting guidelines. J Rehabil Med. 2014;46:385-6.

27. Annals of Surgery. New Instructions for Authors now available. 2015. http:// journals.Iww.com/annalsofsurgery/Blog/FromtheEditorialOffice/pages/post. aspx?PostID=38. Accessed 1 Sept 2015.

28. Roberts J, Shamseer L. Transparency, reproducibility, and validation: raising the quality of reporting in the Journal of Neuro-Ophthalmology. J Neuro-Ophthalmology. 2015;35(1):3-5.

\section{Submit your next manuscript to BioMed Central and we will help you at every step:}

- We accept pre-submission inquiries

- Our selector tool helps you to find the most relevant journal

- We provide round the clock customer support

- Convenient online submission

- Thorough peer review

- Inclusion in PubMed and all major indexing services

- Maximum visibility for your research

Submit your manuscript at www.biomedcentral.com/submit 